\title{
LE VOTE À DISTANCE DES MIGRANTS MEXICAINS: QUEL IMPACT POUR LA DIASPORA DANS LE CHAMP POLITIQUE DU PAYS D'ORIGINE? ${ }^{1}$
}

\author{
Jean-Michel Lafleur*
}

L'élection présidentielle mexicaine de 2006 attiré l'attention des médias du monde entier en raison de la controverse électorale de plusieurs mois ayant suivi ce scrutin qui verra finalement le candidat du parti Partido Acción Nacional l'emporter. Pourtant, au-delà du conflit ayant opposé M. Felipe Calderón Hinojosa (PAN) et M. Andrés Manuel López Obrador (PRD), ces élections ont aussi marqué un tournant dans l'histoire contemporaine du Mexique car elles ont été les premières où la diaspora pouvait participer depuis l'étranger. En raison de la participation d'à peine 32.632 émigrés au dernier scrutin présidentiel, ce premier exercice de droit de vote à distance a néanmoins été caractérisé d'échec par différents observateurs au Mexique et aux Etats-Unis.

Dans cet article, je propose d'envisager en trois étapes les conséquences du droit de vote de la diaspora mexicaine au-delà de la réalité numérique limitée $d u$ dernier scrutin. D'abord, je discuterai de l'évolution du débat sur le droit de vote à distance sur la scène politique mexicaine. Dans cette première partie, je mets donc en lumière les craintes de la classe politique mexicaine à l'égard du poids politique de sa diaspora (particulièrement durant le régime du PRI). Je démontre également comment le processus de transition démocratique entamé à la fin $\mathrm{du}$ $20^{\text {ème }}$ siècle influence les relations entre état et diaspora et ouvre la voie à la réforme sur le droit de vote à distance. Ensuite, après avoir brièvement présenté les données-clés concernant la participation politique de la diaspora en 2006, j'examine deux hypothèses pouvant expliquer la faible mobilisation des Mexicains de l'étranger. D'une part, j'analyse la législation sur le droit de vote à l'extérieur et sa mise en œuvre par les autorités électorales comme des barrières installées délibérément afin de limiter l'impact électoral d'une population sur laquelle les partis politiques ont peu de contrôle. D'autre part, j'émets l'hypothèse, grâce à différentes enquêtes d'opinion et à l'analyse du rôle des associations de migrants dans le processus d'enregistrement des électeurs, que l'intérêt à participer aux élections mexicaines n'est partagé que par une partie de la communauté émigrée. Cet article termine par une discussion sur les évolutions du régime mexicain de vote à l'extérieur au niveau fédéral et régional. Ce faisant, je conclus qu'il est peu probable que l'octroi du droit de vote à la diaspora mexicaine remplit une fonction principalement symbolique pour les autorités mexicaines.

\footnotetext{
1 Papier présenté à la conférence «Etats-Unis Mexique: La frontière au défi de la mondialisation », Institut des Amériques, Paris, 13 octobre 2011.

${ }^{*}$ Chargé de Recherches au Fonds National de la Recherche Scientifique (FRS-FNRS). Chercheur au Centre d'Etudes de l'Ethnicité et des Migrations de l'Université de Liège (Belgique). E-mail: IM.Lafleur@ulg.ac.be.
} 


\section{Le droit de vote à distance des émigrés mexicains : histoire d'un débat}

L'influence de la diaspora mexicaine sur la scène politique du pays d'origine n'est pas un phénomène neuf. Lors de la Révolution Mexicaine de 1910, les émigrés contribue déjà politiquement, financièrement et militairement au destin de leur nation (Gómez Quiñonez, 1983). Malgré les promesses du nouveau régime de prendre en compte les "enfants de la nation» expatriés aux Etats-Unis, l'implication de la diaspora ne donne lieu qu'à une reconnaissance symbolique. Dès lors, aucune politique publique spécifique n'est développée à l'attention des citoyens résidant à l'étranger (Santamaría Gomez, 2003, Delano, 2009).

Les premières traces du débat sur le droit de vote de la diaspora remontent à 1929 lorsqu'un leader de la Révolution -José Vasconcelos- mène une campagne électorale aux Etats-Unis parmi les émigrés en vue de l'élection présidentielle. Pour ce candidat, la diaspora mexicaine devait être considérée comme une population temporairement à l'étranger dont les droits politiques devaient être préservés en attendant leur retour. Bien que sa campagne trouve écho parmi la diaspora, les premières revendications pour le droit de vote depuis l'étranger restent lettre morte après la défaite de Vasconcelos (Santamaría Gómez, 2001).

Le débat sur le droit de vote à distance est né aux alentours de la création du Partido Révolucionario Institucional (PRI). Après la seconde guerre mondiale, ce parti va progressivement asseoir sa domination sur le système politique mexicain en accentuant la caractère présidentiel du régime et en intégrant nombre de ses opposants dans ses rangs. Ce faisant, il met en place un régime corporatiste et modérément autoritaire (Smith, 2003). Malgré ses tentatives de contrôle de la population mexicaine aux Etats-Unis, le régime ne parvient toutefois pas à exporter son modèle corporatiste parmi la diaspora. Pour cette raison, les dirigeants du PRI considèrent le droit de vote à l'extérieur essentiellement comme une menace à l'égard du régime (Calderón Chelius y Martínez Cossío, 2004). Plus tard, l'opposition du PRI au droit de vote de la diaspora sera en outre renforcée par l'intérêt grandissant des autres partis politiques (PRD et PAN) pour l'émigration. Ceux deux partis contribuent d'ailleurs largement au développement d'un discours sur la culpabilité du PRI dans l'émigration massive vers les Etats-Unis.

Pour les émigrés, la question du droit de vote à l'extérieur perd progressivement de sont intérêt à cause du contrôle exercé par le PRI sur le système électoral mexicain. Comme le souligne Martínez Saldaña (1998), le régime du PRI a encouragé l'apathie au sein de la société mexicaine et a rendu l'exercice du droit de vote un simple rituel frauduleux destiné à conforter l'hégémonie du parti dominant. Puisque les élections sous le régime du PRI ne reflétaient plus la volonté populaire, il n'est pas surprenant que la question du droit de vote à distance n'ait pas été une priorité pour les émigrés durant de nombreuses années. Etant donné les craintes du parti dominant et le désintérêt des migrants, il n'est pas surprenant que l'obstacle constitutionnel à l'exercice du droit de vote des émigrés ne soit pas remis en cause durant la majeure partie du $20^{\mathrm{ème}}$ sièclei. 
Il faut attendre les années 80 et le début du processus de transition démocratique au Mexique pour voir la question du droit de vote à distance réapparaitre sur la scène politique mexicaine. Plus généralement, l'on observe un intérêt croissant de la part des autorités pour les questions d'émigration à partir de cette période. Ce changement d'attitude peut être attribué à trois développements domestiques majeurs. Premièrement, des dissidents du PRI crée le Partido de la Revolucion Democratica (PRD) en vue des élections présidentielles de 1988. Dès sa création, ce parti s'organise des deux côtés de la frontière et fait du droit de vote à distance l'une de ses revendications majeures. Deuxièmement, la victoire du candidat du PRI aux élections présidentielles de 1988 est entachée de sérieuses accusations de fraude. Cette situation force le nouveau président Salinas de Gortari à faire de concessions à l'opposition et aux secteurs marginalisés de la société mexicaine. C'est d'ailleurs sous sa présidence que se crée le Programme pour les Communautés Mexicaines à l'Etranger (connu sous le nom de PCME en espagnol) (Goldring, 1998, Roberts, et al., 1999). En ce qui concerne la question du droit de vote, le PRD profite du conflit au Chiapas pour négocier avec le PRI l'abolition de l'article 36 de la Constitution empêchant aux migrants de voter s'ils ne sont pas présents dans leur circonscription électorale le jour de l'élection. Le droit de vote à distance reste toutefois impossible dans la mesure où il manque toujours une législation réglementant l'exercice de ce droit. Troisièmement, le souhait du nouveau gouvernement mexicain de rejoindre la zone de libre-échange nord-américaine (ALENA) l'encourage à courtiser la communauté mexicano-américaine. Les autorités mexicaines espéraient effectivement que, à l'image d'autres lobbies ethniques, cette communauté fasse pression en faveur de leur pays d'origine auprès du Congrès des Etats-Unis (de la Garza y Desipio, 1998, Leiken, 2000).

Durant cette période de transition démocratique s'étalant des années 1980 à l'élection présidentielle de 2000, deux acteurs institutionnels voient également leurs pouvoirs renforcés. Ils utilisent cette influence pour peser sur le débat sur le droit de vote à distance. La première institution est l'Institut électoral fédéral (IFE) créé après l'élection de 1988 afin de mettre un terme aux fraudes électorales. L'IFE est aussi chargé en 1996 d'étudier les modalités selon lesquelles les autorités mexicaines pourraient mettre en œuvre le droit de vote à distance. A cette fin, l'IFE met sur pied un comité d'experts qui conclut en 1998 qu'il serait techniquement possible pour les autorités mexicaines de faire participer les émigrés à l'élection présidentielle de 2000.

Le second acteur est la région (estado) qui se voit attribuée de nouvelles responsabilités administratives et fiscales. En raison de leur plus forte proximité avec les familles de migrants restés au pays, nombre d'élus régionaux (et municipaux) vont développer un intérêt particulier pour la question de l'émigration. C'est d'ailleurs au niveau régional que les premières avancées en terme de participation politique à distance sont enregistrées. En effet, l'état de Zacatecas est le premier à adopter le principe de "résidence binationale » permettant aux émigrés à se présenter comme candidats aux élections municipales et régionales dans cet état (Moctezuma Longoria, 2003, Bakker y Smith, 2003). Les prérogatives des régions dans le domaine de l'émigration augmentant, elles deviennent des cibles privilégiées des associations d'émigrés 
tentant de rallier des élus mexicains à la cause du droit de vote à distance. Ces associations pressent donc des politiciens régionaux influents dans l'espoir que ceux-ci relaient la revendication pour le droit de vote à distance auprès des autorités fédérales.

Face aux développements démocratiques se produisant dans leur pays d'origine, la demande des migrants à voter dans le pays d'origine va progressivement réapparaitre. Pour des associations établies aux Etats-Unis comme l'Asemblea Mexicana por el Sufragio en el Exterior (AMSE) ou le Concilio Hispano, le droit de vote devient à nouveau une priorité à la fin des années 80 . Elles considèrent d'ailleurs que la transition vers la démocratise est incomplète tant que la diaspora n'a pas le droit de vote (Lafleur y Calderón Chelius, 2011). Pour se faire entendre, ces associations font bien entendu pression sur les partis politiques mexicains mais organisent aussi des élections symboliques devant les consulats mexicains lors des élections présidentielles de 1988 et 1994 (Sepúlveda, 1991). Développer le rôle des associations

Malgré l'émergence d'une contexte politique favorable aux droits des émigrés décrit ci-dessus, le Parlement mexicain décide finalement de ne pas permettre le droit de vote à distance pour les élections présidentielles de 2000. Le PRI s'oppose en effet à toute tentative du PAN ou du PRD d'introduire ce nouveau droit dans la législation mexicaine arguant tantôt des contraintes techniques et tantôt du caractère illégitime du droit de vote des citoyens non-résidents. Toutefois, les émigrés sont autorisés à voter dans un nombre limité de bureaux de vote situé dans des villes mexicaines frontalières. En raison des restrictions liées à cet exercice, seul un nombre très limité de migrants se déplacera le jour du vote pour participer au scrutin présidentiel (Espinoza Valle, 2004).

C'est en franchissant une nouvelle étape dans la transition vers la démocratie que le débat sur le droit de vote à distance va enfin connaître un dénouement heureux pour la diaspora. L'élection du Président Vicente Fox Quesada, premier président non-issu du PRI depuis 71 ans, fait entrer les relations entre Etat et diaspora dans une nouvelle ère. Sous le PRI, les émigrés étaient traditionnellement considérés comme des citoyens de seconde classeii. Durant les dernières années du régime, la croissance exponentielle des remises d'épargne et la pression de l'opposition avaient certes encouragé le gouvernement à développer une série de politiques d'attention à leur égard. Néanmoins, avec l'élection de M. Fox Quesada à la présidence, le paradigme change et les migrants sont désormais reconnus comme " héros de la nation mexicaine ». En tant qu'ancien gouverneur de l'état de Guanajuato, Fox avait compris depuis longtemps l'importance de la question migratoire dans la politique mexicaine. Lors de sa campagne électorale en 2000, il se rend d'ailleurs à plusieurs reprises aux Etats-Unis et se fait remarquer en distribuant des cartes téléphoniques aux migrants dans l'espoir que ceux-ci appellent leurs proches et leur recommandent de voter Fox. Dès son investiture, le Président Fox fait des liens avec la diaspora une priorité et promet que le droit de vote à distance sera une réalité avant la fin de son mandat. Cet engagement, pris dans un contexte de compétition croissante entre les partis politiques, stimule bien entendu l'intérêt 
des associations d'émigrés qui redoublent d'effort dans leurs campagnes de pression sur les partis à partir de 2001 .

La Coalition pour les Droits Politiques des Mexicains à l'Etranger (CDPME selon son acronyme espagnol) joue un rôle particulièrement important durant les années Fox. Ce groupe de pression, va, en effet, mettre sur pied une stratégie habile pour faire avancer le débat sur le droit de vote à distance. En rassemblant des représentants d'associations de migrants et des lobbyistes établis à Mexico, la CDPME bénéficie à la fois du soutien des communautés émigrées et de l'expertise d'intellectuels mexicains défendant leurs intérêts auprès du Parlement mexicain. Cette alliance confère à la CDPME une grande légitimité dans ses contacts avec les partis politiques mexicains. La CDPME utilisera donc ce crédit pour contraindre les partis politiques à se positionner clairement sur le droit à distance en les menaçant d'être présenté aux Etats-Unis comme les ennemis de la diaspora s'ils ne soutenaient la réforme.

Malgré la pression de la CDPME et d'autres associations, le Parlement mexicain reste divisé sur la question. En effet, chaque parti se positionne sur de droit de vote à distance en fonction de l'impact attendu sur les résultats électorats d'une diaspora représentant plusieurs millions d'électeurs potentiels. Pour les raisons évoquées plus haut, le PRI craint un vote massif de la diaspora qui lui serait défavorable. Le PRD, en revanche, est persuadé du soutien de la diaspora. Certains de ses membres adoptent donc une position maximaliste refusant toute réforme qui n'inclurait pas une représentation parlementaire garantie pour les émigrés. Le parti du Président Fox - le PAN — est quant à lui divisé en raison du fait que les émigrés ne correspondent pas à son électorat traditionnel de classe moyenne.

Face à l'indécision des partis politique et à l'incertitude quant à l'impact réel de la diaspora sur les résultats électoraux, le Président Fox charge son vice-ministre de l'Intérieur de consulter les partis politiques. Le 6 Avril 2004, l'ensemble de partis politiques s'accordent sur des conditions acceptables pour tous pour l'exercice du droit de vote à distance. Ce compromis, qui formera ensuite le cœur de la législation du 28 Juin 2005 sur le droit de vote à distance, repose entièrement sur la limitation de l'impact du vote de la diaspora sur les résultats électoraux. Pour ce faire, les partis s'entendent pour 1) limiter le droit de vote à distance aux élections présidentielles, 2) empêcher toute forme de représentation parlementaire au niveau fédéral, 3) permettre le droit de vote à distance par courrier postal, 4) interdire toute forme de campagne électorale à l'étranger, 5) limiter le droit de vote aux citoyens possédant une carte d'électeur, et 6) interdire la délivrance de carte d'électeurs à l'étranger. Sachant que bon nombre de Mexicains aux Etats-Unis ne possèdent pas de carte d'électeur et qu'il est peu vraisemblable que des sans-papiers prennent le risque de traverser la frontière pour l'obtenir cette carte, cet accord garantissait que seul un nombre limité d'émigré allait pouvoir participer. Un an à peine avant les élections présidentielles de 2006, il restait désormais aux autorités électorales à mettre en œuvre la nouvelle législation à l'étranger. Pour les associations de migrants, un cap historique est franchi avec la reconnaissance formelle du droit de vote à 
distance. Cet enthousiasme va toutefois rapidement disparaître avec l'enregistrement d'un nombre extrêmement limité d'électeurs à l'étranger.

\section{La participation des émigrés à l'élection présidentielle de 2006}

Afin de déterminer en quoi le droit de vote à distance a changé les relations entre le Mexique et sa diaspora, il est nécessaire de présenter quelques données chiffrées concernant le scrutin de 2006. Comme je l'ai indiqué plus haut, nombre de politiciens mexicains ont longtemps refusé de soutenir le droit de vote à distance car ils craignaient l'impact de ces électeurs sur les résultats électoraux de leur parti. En 1998, un comité d'experts mis en place par l'Institut Electoral Fédéral (IFE) estimait, en effet, qu'environ 11 millions d'émigrés étaient en âge de voter(IFE, 1998). Peu avant l'adoption de la législation de 2005, l'IFE revoit toutefois son estimation en indiquant qu'environ 4 millions d'émigrés possèdent l'indispensable carte d'électeur pour pouvoir s'enregistrer à l'étranger. L'IFE avertit également que nombre de facteurs pourraient affecter le taux de participation au final (IFE, 1998). Parmi ces facteurs, l'IFE note qu'il est probable que nombre de migrants non documentés se soient débarrassés de tout document d'identité avant le passage de la frontière. Face à l'incertitude concernant la mobilisation de la diaspora mexicaine, les associations de migrants comme la CDPME considéraient que la participation de 400.000 électeurs serait un succès pour le premier exercice de droit de vote à distance. Marcelli et Cornelius (2005) soulignèrent quant à eux qu'il existe généralement une certain apathie parmi les émigrés lorsqu'il s'agit de voter pour les élections d'un pays où ils ne résident plus. Pour cette même raison, ils estimèrent que seuls 125.000 à 360,000 émigrés feraient au final l'effort de s'enregistrer comme électeur.

Ces différentes estimations vont toutefois largement dépasser le nombre réel d'électeurs inscrits entre le $1^{\mathrm{er}}$ octobre 2005 et le 15 janvier 2006. En effet, seuls 54.866 émigrés ont envoyé le formulaire d'inscription comme électeur à l'étranger aux autorités électorales mexicaines. Ces dernières ont ensuite rejeté une série de demandes jugées non-conformes et ont retenu enfin 40.876 électeurs à l'étranger auxquels ils ont envoyé un bulletin de vote par voie postale. Au final, 81\% de ces électeurs enregistrés - soit 32,632 personnes ont participé au scrutin en retournant leur bulletin aux autorités électorales également par voie postale. Bien que certains chercheurs aient déjà démontré qu'il soit fréquent que moins de $5 \%$ des émigrés utilisent leur droit de vote à distance dans les pays qui l'autorise (Calderón Chelius, 2003), le cas mexicain frappe toutefois par l'extrême faiblesse du taux d'enregistrement au regard de la taille de l'émigration mexicaine et de ses liens politiques, sociaux et économiques avec le pays d'origine. Avant de discuter en détails des raisons qui ont conduits à une si faible participation électorale à l'étranger, trois éléments méritent d'être mis en valeur.

Tout d'abord, bien que la population mexicaine aux Etats-Unis représente 98\% de l'ensemble de la population mexicaine à l'étranger, les émigrés vivant dans ce pays représentent seulement $85,6 \%$ des électeurs ayant participé au scrutin depuis l'étranger. Dans le tableau 1, on constate effectivement que dans d'autres 
pays ayant des populations mexicaines bien plus réduites, la proportion d'électeurs enregistrés par rapport à l'ensemble de la population mexicaine établie dans ce pays est bien plus élevée. Différentes raisons présentées plus bas peuvent expliquer le plus fort taux d'enregistrement parmi différentes communautés mexicaines établies en dehors des Etat-Unis.

Tableau 1. Nombre de demande d'enregistrement comme électeur par pays de résidence

\begin{tabular}{|l|l|c|c|}
\hline & Pays de résidence & $\begin{array}{c}\text { Nombre de demandes } \\
\text { d'enregistrement }\end{array}$ & $\begin{array}{l}\text { Population mexicaine } \\
\text { totale }\end{array}$ \\
\hline $\mathbf{1}$ & Etats-Unis & 35.763 & 11.500 .000 \\
\hline $\mathbf{2}$ & Espagne & 1.238 & 21.007 \\
\hline $\mathbf{3}$ & Canada & 863 & 49.925 \\
\hline $\mathbf{4}$ & France & 510 & 1.393 \\
\hline $\mathbf{5}$ & Royaume Uni & 447 & 5.297 \\
\hline $\mathbf{6}$ & Allemagne & 393 & 9.225 \\
\hline $\mathbf{7}$ & Italie & 212 & 6.798 \\
\hline $\mathbf{8}$ & Suisse & 188 & 751 \\
\hline $\mathbf{9}$ & Pays-Bas & 100 & 1.338 \\
\hline $\mathbf{1 0}$ & Belgique & 83 & - \\
\hline $\mathbf{1 1}$ & Autres pays & 1079 & \\
\hline
\end{tabular}

Source : (COVE, 2006, IME, 2010)

Ensuite, la population d'électeurs mexicains émigrés se démarque par le fait les citoyens issus des états à forte tradition d'émigration comme le Zacatecas y sont peu représentés. Pourtant, comme nous l'avons vu, le Zacatecas est un état pionnier dans le développement des droits politiques des citoyens établis à l'étranger. En revanche, les états de plus récente émigration que sont le District Fédéral et l'état de Mexico ont enregistré nombre d'électeurs. Au total, la moitié des électeurs inscrits sont originaires de seulement 5 états.

Tableau 2. Etat d’origine des émigrés inscrits au registre des électeurs

\begin{tabular}{|l|l|l|}
\hline & Région d'origine & Nombre de demandes \\
\hline $\mathbf{1}$ & District fédéral & 6.281 \\
\hline $\mathbf{2}$ & Jalisco & 5.047 \\
\hline $\mathbf{3}$ & Etat de Mexico & 4.149 \\
\hline $\mathbf{4}$ & Michoacán & 3.368 \\
\hline $\mathbf{5}$ & Guanajuato & 2.793 \\
\hline $\mathbf{6}$ & Nuevo Leon & 1.799 \\
\hline $\mathbf{7}$ & Puebla & 1.631 \\
\hline $\mathbf{8}$ & Baja California Norte & 1.582 \\
\hline $\mathbf{9}$ & Chihuahua & 1.235 \\
\hline $\mathbf{1 0}$ & Veracruz & 1.191 \\
\hline
\end{tabular}




\begin{tabular}{|l|l|l|}
\hline $\mathbf{1 1}$ & Autres & 11.800 \\
\hline
\end{tabular}

Source : (COVE, 2006)

Enfin, le dernier élément notable dans les données sur la participation politique concerne les résultats électoraux. Dans le tableau 3, on constate que l'orientation politique des Mexicains de l'étranger est sensiblement différente de celle des électeurs résidant sur le territoire national. Contrairement aux préjugés ayant dominé le débat sur l'octroi du droit de vote à distance, le PRD n'a pas été le principal récipiendaire de voix issues de l'émigration. Le PAN, conformément à l'intuition du Président Fox convaincu du potentiel électoral de l'émigration pour son parti, a quant à lui largement dominé l'élection présidentielle à l'étranger. Le PRI, qui s'est longtemps opposé au droit de vote à distance, a en revanche obtenu la confirmation qu'il possède peu de soutien parmi les émigrés. Etant donné le nombre limité d'émigrés ayant participé à l'élection, ces résultats n'ont toutefois pas une valeur représentative de l'opinion politique de l'ensemble des émigrés mexicains. En effet, comme nous le verrons plus bas, le processus d'enregistrement a introduit une série de biais en défaveur des émigrés les plus marginalisés. McCann et ses collègues (2006) ont en effet démontré que les émigrés mexicains aux Etats-Unis, dans leur ensemble, n'ont pas préférences politiques sensiblement différentes de celles des électeurs non-migrants. En menant une enquête auprès des émigrés mexicains enregistrés ou non, ils ont démontré que l'opinion politique de l'ensemble de cette population est proche de ces des électeurs résidant sur le territoire national. Sachant que l'élection présidentielle mexicaine de 2006 fut très disputée et que moins de 250.000 voix ont séparé le candidat du PAN de celui du PRD, ce débat sur l'opinion politique n'est pas sans intérêt pour les partis politiques si le taux de participation devait être plus important lors des présidentielles de 2012.

Tableau 3. Résultats de l'élection présidentielle mexicaine de 2006

\begin{tabular}{|l|c|c|c|}
\hline \multicolumn{1}{|c|}{ Parti } & $\begin{array}{c}\text { Résultats électoraux } \\
\text { parmi les émigrés }\end{array}$ & $\begin{array}{c}\text { Préférences électorales } \\
\text { des émigrés observées } \\
\text { par McCann et al. (2006) }\end{array}$ & $\begin{array}{c}\text { Résultats globaux de } \\
\text { l'élection présidentielle } \\
\text { mexicaine }\end{array}$ \\
\hline PAN & $58,29 \%$ & $37 \%$ & $35,89 \%$ \\
\hline PRD & $34,00 \%$ & $34 \%$ & $35,31 \%$ \\
\hline PRI & $4,17 \%$ & $21 \%$ & $22,26 \%$ \\
\hline
\end{tabular}

Source : (McCann, et al., 2006, COVE, 2006)

\section{Comment expliquer la participation limitée des émigrés à l'élection présidentielle de 2006 ?}

Lorsque les données sur l'enregistrement des électeurs ont été révélées et avant même que les électeurs enregistrés fassent usage de leur droit, une polémique a éclate entre les représentants de l'IFE et diverses associations de migrants. Pour ces dernières, les autorités électorales n'ont pas fait suffisamment d'efforts pour enregistrer les électeurs à l'étranger. Elles considèrent donc que la faible participation des émigrés est due à une législation trop restrcitive et à sa mise en œuvre déficiente par les autorités électorales. L'IFE, en revanche, estime qu'il a mis en œuvre nombre d'initiatives pour encourager les électeurs à s'enregistrer 
dans les limites du mandat qui lui était accordé par la législation. Pour certains fonctionnaires de l'IFE, comme pour certains politiciens mexicains opposés au droit de vote à distance, le faible taux d'enregistrement des électeurs à l'étranger n'est donc que le reflet du manque d'intérêt de cette population pour la politique de leur pays d'origine. Dans cette troisième partie, je m'examine la validité de ces deux hypothèses.

3.1. « La lourdeur administrative du processus d'enregistrement a découragé les émigrés de participer »

Comme nous l'avons vu dans la première partie de l'article, la législation mexicaine sur le droit de vote à distance est le résultat d'un compromis entre les différents partis politiques. Au cœur de ce compromis, on retrouve le souhait partagé par l'ensemble de partis (à l'exception de certains membres du PRD) que les électeurs résidant à l'étranger ne soit pas en mesure d'avoir un poids décisif sur le résultat de l'élection présidentielle. Pour y parvenir, le PAN, le PRI et le PRD se sont accordés pour limiter le droit de vote aux émigrés possédant une carte d'électeur, pour rendre impossible la délivrance de ce document à l'étranger et pour interdire tout acte de campagne électorale à l'étranger. Malgré l'accord des partis dès 2004 sur ces différentes restrictions au droit de vote à distance, il faudra encore une année au Parlement pour adopter la législation. Différentes disposition de la loi du 28 juin 2005 confirmeront la volonté des partis de limiter la participation électorale à l'étranger.

Tout d'abord la législation charge l'IFE de l'ensemble des opérations électorales à l'extérieur. Ce choix se justifie par le fait que, depuis sa création, l'IFE est considéré comme le garant de la légitimité du processus électoral. Comme l'IFE a été pour empêcher l'immixtion du pouvoir exécutif, il était peu concevable pour le législateur d'autoriser le Secrétariat des Affaires Etrangères à organiser le processus électoral à l'étranger. Pourtant, en raison de sa densité et de ses contacts privilégiés avec la population mexicaine à l'étranger, le réseau consulaire mexicain aurait pu être un allié de choix pour l'IFE dans sa campagne d'enregistrement à l'extérieur. Au lieu de cela, l'interdiction faite aux consulats empêche même ceux-ci de communiquer à l'IFE les adresses d'électeurs potentiels. Après l'adoption de la loi sur le droit de vote à distance, l'IFE s'est donc retrouvé seul pour développer en mois une stratégie d'enregistrement de plusieurs millions d'électeurs potentiels avec lesquels elle ne possédait jusqu'alors aucun relais et résidant en dehors du territoire national.

Ensuite, la procédure d'enregistrement réglée par l'article 275 de la loi électorale n'autorise à s'enregistrer comme électeur que les émigrés déjà en possession d'une carte d'électeurs. Comme indiqué précédemment, cette restriction est un élément-clé dans la tentative des partis politiques de limiter l'impact des électeurs émigrés sur le résultat de l'élection. En effet, en obligeant les émigrés intéressés à s'enregistrer à rentrer au pays pour obtenir une carte d'électeur, il était évident que les millions d'émigrés qui ne possédaient pas cette carte ne voteraient pas (nombre d'entre eux sont en situation irrégulière aux Etats-Unis et ils ne vont donc pas courir pas le risque de traverser la frontière pour l'obtenir). 
Pour suivre, la législation électorale prévoit que les électeurs doivent s'enregistrer au moyen d'un formulaire mis à disposition par l'IFE. Les émigrés souhaitant voter doivent retourner ce formulaire par courrier recommandé à l'IFE en l'accompagnant d'une copie de leur carte d'électeur et d'une preuve de leur résidence à l'étranger (ex. une facture d'électricité). Ces dispositions ont été critiquées par les associations de migrants pour différentes raisons. L'IFE s'étant, dans un premier temps, essentiellement sur son site internet pour fournir le formulaire d'enregistrement, il est évident que les migrants moins éduqués ont dès lors été pénalisés. Ces mêmes migrants ont également été pénalisés par le fait qu'il était nécessaire de savoir lire et écrire pour s'enregistrer (par comparaison, un enregistrement au consulat évite cette difficulté). L'obligation de renvoyer le formulaire par courrier recommandé a également été critiquée en raison de son coût (environ 8 dollars US) et du fait qu'il oblige le migrant à se déplacer au bureau de poste. Ces différents éléments confortent l'existence d'un biais dans le processus d'enregistrement en faveur des émigrés plus éduqués et moins marginalisés d'un point de vue socio-économique.

Enfin, l'IFE a été accusé par les associations de migrants d'avoir mené une trop faible campagne d'information à l'étranger et d'avoir fait une interprétation restrictive de la loi électorale. Comme j'ai eu l'occasion de le dire, le législateur mexicain a laissé peu de temps à l'IFE pour mettre en œuvre une stratégie d'enregistrement des émigrés. Pour cette raison, l'IFE s'est principalement contentée d'informer la diaspora par l'intermédiaire de son site internet, des médias et de l'affichage dans les lieux publics fréquentés par les Mexicains aux Etats-Unis. C'est seulement quelques semaines après le début du processus d'enregistrement, alors que très peu de demandes lui était parvenues, que l'IFE a décidé d'adapter sa stratégie en s'entourant de leaders d'associations de migrants. Ces derniers ont alors insisté pour, qu'au-delà d'une simple campagne d'information, l'IFE soit présent dans les quartiers à forte présence mexicaine aux Etats-Unis afin d'aller à la rencontre directe des électeurs pour les aider à s'enregistrer. Cette réponse tardive de l'IFE associée au fait qu'il a rejetté plus de 10.000 formulaires d'inscriptions jugés non-conformes, a incité nombre de migrants à percevoir l'IFE comme un obstacle dans la concrétisation du droit de vote à distance qu'ils espéraient pouvoir exercer depuis si longtemps.

En conclusion, malgré les critiques répétées des migrants, il faut noter que l'IFE a été placée dans une position schizophrénique puisqu'elle devait à la fois veiller à ce que les partis ne fassent pas campagne électorale à l'étranger où l'IFE n'a pas de juridiction et distribuer la propagande électorale auprès des émigrés ayant réussi à s'enregistrer. Cette dernière mission s'avérait d'autant plus inconfortable pour l'IFE qu'il fut uniquement créé dans le but de stimuler la participation électorale et d'assurer que le processus électoral soit mis en œuvre dans le respect de la loi. A la lecture de cet objectif, l'on comprend également que le mécontentement des migrants à l'égard de l'IFE apparaît injustifié aux yeux de l'institution. Pour cette dernière, la première expérience de vote à distance est un succès dans la mesure où elle a déployé une large campagne d'information et la légalité du processus électoral a l'étranger n'a pas souffert de contestation. Ces leaders de l'émigration mexicaine ayant fondé leur lutte pour le droit de vote 
sur l'idée que l'émigré souhaitait continuer à influer sur le destin de son pays d'origine malgré la distance, le faible taux d'enregistrement apparaissait aussi comme un désaveu. En effet, malgré les barrières administratives et la frilosité de l'IFE, l'enregistrement de 40,000 électeurs invite également à s'interroger sur l'intérêt réel des émigrés à voter à distance.

3.2. «Les émigrés ne sont pas intéressés à participer aux élections dans leur pays d'origine»

La question de l'intérêt des diasporas pour la politique du pays d'origine n'est pas neuve. Depuis plusieurs décennies, des chercheurs s'intéressent au nationalisme de longue-distance (Anderson, 1992), à l'implication des réfugiés dans les conflits du pays d'origine (Turner, 2008) et au lobbying des communautés immigrées sur les autorités du pays d'accueil en faveur de leur pays d'origine (Smith, 2000, Østergaard-Nielsen, 2003, Lafleur, 2005). La question de l'intérêt des migrants pour le vote à distance reste toutefois une question peu abordée dans la littérature scientifique. Dans le cas du Mexique, il nous semble toutefois que la question de l'intérêt des migrants doit être envisagé $\mathrm{du}$ point de vue individuel mais également du point de vue des associations d'émigrés dont la mobilisation ou non en faveur de la participation de leurs membres est révélatrice de l'importance que la communauté accorde au vote à distance.

$\mathrm{Au}$ niveau individuel, la littérature en science politique sur la participation électorale a traditionnellement mis l'accent sur les facteurs socio-économiques (niveau d'éducation, emploi...), institutionnels (type d'élection, mode de scrutin...) et politique (caractère compétitif de l'élection, importance perçue par l'électeur) comme variables explicatives pour le taux de participation des électeurs (Geys, 2006). En ce qui concerne la participation politique à distance, seuls quelques travaux récents ont tenté de mesurer les facteurs influençant la participation des émigrés aux élections dans leur pays d'origine (Lafleur, 2012, Escobar, 2011). Dans ce cas particulier, il semble nécessaire d'opérer une distinction entre intérêt pour la politique du pays d'origine et motivation à s'enregistrer comme électeur.

Dans leur enquête conduite auprès de 1000 émigrés Mexicains aux Etats-Unis entre février et juin 2006, McCann et ses collègues (2006) ont trouvé qu'entre 8 et 12 de la diaspora a suivi avec grande attention la campagne électorale mexicaine de 2006. Suro et Escobar (2006)se sont quant à eux intéressés au niveau d'information des émigrés quant au processus d'enregistrement à l'étranger. En interviewant 1000 émigrés interviewés juste après la fin du processus d'enregistrement à l'étranger. Bien que 78\% d'entre eux étaient au courant que la diaspora pouvait participer au scrutin de 2006, seuls $36 \%$ savaient que la date limite d'inscription dans le registre électoral était déjà passée. Interrogés sur les raisons de leur non-inscription comme électeurs, $67 \%$ ont reconnus ne pas posséder les documents nécessaires, $55 \%$ se sont déclarés mal informés sur le processus d'enregistrement et $46 \%$ ont trouvé a procédure 
trop complexe. Seuls $2 \%$ ont déclaré ne pas s'être enregistrés par manque d'intérêt pour la politique mexicaine.

Les données de ces deux enquêtes permettent de conclure que le potentiel de participation électorale à distance est bien plus large que les 40.000 électeurs inscrits en 2006. Cet élément tend à conforter l'idée que les restrictions bureaucratiques au droit de vote ont découragés nombre d'électeurs potentiels. Comme l'a démontrée l'enquête réalisée en 1998 par Espinoza Valle (2004) auprès d'émigrés résidant proches de la frontière, 75\% d'entre eux ne sont pas disposés à passer plus d'une heure en démarches administratives afin de s'enregistrer comme électeur. Au contraire, comme le souligne Waldinger (2008), deux millions d'émigrés Mexicains étaient disposés à faire de longues files pour obtenir une carte d'identité consulaire mexicaine entre 2002 et 2003 car ils considèrent que ce un document peut faciliter leur vie quotidienne aux Etats-Unis. Ce dernier élément démontre que, même si l'intérêt pour la politique du pays d'origine est réel parmi les émigrés, les migrants sont disposés à faire un nombre limité d'efforts afin de pouvoir exercer leur droit de vote à distance.

L'importance des barrières administratives dans la décision de s'enregistrer étant confirmée, il est nécessaire de s'interroger également sur le rôle des associations d'émigrés pour aider la diaspora à les franchir. En raison de leurs liens avec les émigrés, ces associations sont, plus que tout autre acteur, en mesure de diffuser l'information sur le processus électoral et d'aider les migrants à réaliser un processus d'inscription jugé complexe. Toutefois, comme le confirme les entretiens réalisés avec différentes associations, leur rôle s'est souvent limité à la diffusion de brochures informatives ou de formulaires d'inscription auprès de leurs membres. Les associations elles-mêmes reconnaissent que cette stratégie a une influence limitée sur les émigrés. Elles estiment en revanche qu'une aide personnalisée aux migrants pour compléter leur formulaire d'inscription permet de dépasser les barrières administratives. Cette stratégie n'a été mise en place que par un nombre limité d'associations et à un stade tardif du processus d'enregistrement. Trois éléments permettent de comprendre l'implication limitée des associations.

Tout d'abord, pour les associations ayant lutté pour le droit de vote à distance, le passage de la loi en 2005 a marqué la fin d'un combat. Avec l'octroi du droit de votes, ces dernières considéraient donc le vote était acquis et qu'il revenait maintenant aux autorités de le mettre en œuvre. Pour cette raison, certaines associations ont opté pour une attitude attentiste durant le processus d'enregistrement jusqu'à ce qu'ils se rendent compte que la participation allait être extrêmement limitée. Ensuite, comme l'a souligné un membre de la CDPME durant l'entretien, le faible taux d'enregistrement des électeurs à l'étranger invite également à repenser la nature du lien entres les associations et les migrants. De l'aveu même de cette personne, si une association prétend représenter plusieurs centaines de milliers de Mexicains aux Etats-Unis et décide de soutenir le droit de vote à distance, comment expliquer qu'elle n'arrive pas à convaincre $1 \%$ de ses membres à s'enregistrer ? Cet élément nous rappelle que la participation électorale est guidée par une multitude de facteurs et que les injonctions des élites de l'émigration n'ont qu'une influence limitée sur la 
décision finale du migrant à faire ces démarches. Enfin, une partie importante des associations mexicaines développées aux Etats-Unis durant les deux dernières - sont connues sous le nom de hometown associations- ne sont réellement en mesure de participer à la promotion du droit de vote à distance. D'une part, ces associations se concentrent sur des activités de développement dans leurs communautés d'origine au Mexique. D'autre part, leur structure de petite et leur orientation vers le pays d'origine les rends peu appropriées pour des actions civiques dans le pays de résidence.

$\mathrm{Au}$ final, on peut conclure que les associations ont été relativement attentistes dans le processus d'enregistrement a été relativement limité. Face à la taille de la diaspora mexicaine aux Etats-Unis et à la difficulté d'entrer en contact avec cette population souvent marginalisée, elles avaient pourtant la possibilité d'aider l'IFE dans le processus d'enregistrement. En effet, à l'image de la petite communauté mexicaine en Suisse où une association de migrants est entrée avec virtuellement l'ensemble des migrants résidant dans ce pays, l'implication des associations dans peut faire la différence.

\section{Conclusion: Quel impact pour la diaspora dans le champ politique mexicain?}

Dans la première partie de cet article, j'ai présenté les conditions dans lesquelles le débat au sein de la classe politique mexicaine sur le droit de vote à distance a évolué du début du 20ème siècle jusqu'à l'adoption de la réforme en 2005. En mettant en lumière l'importance croissante des relations avec la diaspora dans un contexte de transition démocratique, j'ai démontré que les autorités mexicaines ont progressivement changé leur image de l'émigré en tant que citoyen de seconde classe à celle de nouveau héro de la nation mexicaine. Le développement de politiques d'attention à destination des émigrés a fait du Mexique un exemple pour nombre d'états (grâce notamment à sa politique d'attraction des remises, à la création d'un conseil consultatif de l'émigration et au développement d'un réseau consulaire extrêmement dense).

En ce qui concerne le droit de vote à distance, ce sont les préoccupations des parties politiques quant à l'impact du vote de la diaspora plus que toute autre considération qui ont conditionnées l'adoption de la législation. En effet, c'est la crainte de voir les millions de Mexicains établis à l'étranger déterminer le résultat de l'élection présidentielle qui a dicté l'adoption d'une série de restrictions administratives permettant de limiter le nombre d'électeurs tout en assurant la régularité du processus électoral à l'étranger. Ces restrictions ont permis de limiter avec succès l'impact du vote de la diaspora alors que l'élection de 2006 s'était jouée à quelques 250.000 voix entre les candidats du PAN et du PRD.

Ces restrictions administratives au droit de vote à distance ont également eu pour conséquence de rendre la participation électorale de la diaspora purement symbolique. Avec l'arrivée au pouvoir du Président Fox en 2001 -premier président issu d'un autre parti que le PRI en 71 ans- le droit de vote à distance 
est devenu un outil pour signifier aux émigrés qu'ils appartiennent toujours à la nation en dépit de la distance. A part pour quelques militants du PRD revendiquant avec force une représentation parlementaire pour l'émigration mexicaine, l'idée des partis mexicains n'a donc jamais été de permettre aux 12 millions de migrants mexicains de redéfinir l'équilibre électoral dans leur pays d'origine. Pour cette raison, comme le souligne Smith (2008), l'adoption de la législation de 2005 n'a pas nécessairement bénéficié à la diaspora. Avant, l'émigration mexicaine n'avait pas de droit de vote mais une influence considérable sur les candidats venant faire campagne aux Etats-Unis. Aujourd'hui, la diaspora possède le droit de vote mais son influence est limitée par les restrictions à l'enregistrement et par l'interdiction faite aux partis de faire campagne à l'étranger.

Dans la seconde partie de l'article, je me suis attardé sur les raisons de l'abstention en me concentrant sur les barrières administratives d'une part et sur l'intérêt des migrants pour la politique du pays d'origine d'autre part. Comme le démontre les exemples d'autres nations autorisant le droit de vote à distance, les règles administratives encadrant le droit de vote à distance ont une influence considérable sur la participation électorale. L'Italie, par exemple, invite tous les émigrés enregistrés dans le registre consulaire à voter en leur envoyant à domicile les bulletins de vote sans demande d'enregistrement préalable. Ce mécanisme d'inscription automatique et de vote par correspondance permet à l'Italie d'enregistrer la participation de plus d'un million de citoyens dispersés sur l'ensemble de la planète. Il a aussi ouvert la voie à plusieurs cas de fraudes avérés. En Bolivie, le législateur a opté pour un mode d'enregistrement similaire $\mathrm{au}$ système mexicain. En adoptant une démarche plus proactive dans ses contacts avec les communautés émigrées, les autorités électorales ont néanmoins réussi à enregistrer à enregistrer 169,000 électeurs en 45 jours.

$\mathrm{Au}$ Mexique, la préoccupation de l'IFE de garantir des élections régulières l'a conduit à adopte une attitude extrêmement prudente dans le déroulement des opérations électorales à l'étranger. A la suite de l'élection de 2006 et du mécontentement de certaines associations d'émigrés, l'IFE a entamé une réflexion sur les possibilités d'améliorer la participation électorale à l'étranger à l'avenir. Cette réflexion est toutefois limitée par le cadre législatif existant qu'aucun parti politique mexicain n'est disposé à changer par crainte qu'il n'influence de façon décisive l'élection présidentielle de 2012. Pour cette raison, l'IFE n'a pu prendre des mesures d'ajustement comme l'organisation de campagnes d'information pour encourager les migrants à demander une carte d'électeurs lorsqu'ils rentrent en visite au Mexique. De même, afin de pouvoir compter sur l'appui des associations dans le processus d'enregistrement, l'IFE a mis sur pied un réseau de coordination avec celles-ci. Ce réseau devrait permettre une meilleure diffusion de l'information sur le processus d'enregistrement au sein des communautés mexicaines. Toutefois, bien que ces initiatives devraient permettre à l'IFE d'enregistrer un nombre supérieur d'électeurs à l'étranger en vue des élections de 2012, seule une modification substantielle du cadre législatif à moyen ou long terme sera en mesure de rendre la participation électorale de la diaspora autre chose qu'une reconnaissance symbolique du lien unissant le Mexique à sa diaspora. 


\section{Bibliographie}

Anderson, B. R. "Long-distance Nationalism. World Capitalism and the Rise of Identity Politics". (1992). [Consultada el Access 1992]

Bakker, Matt; Smith, Michael P. "El Rey del Tomate: Migrant Political Transnationalism and Democratization in Mexico". Migraciones Internacionales. Vol. 2. No. 1 (2003). P. 59-83.

Calderón Chelius, Leticia. "Votar en la distancia". (2003). [Consultada el Access 2003]

Calderón Chelius, Leticia; Martínez Cossío, Nayamín. "La democracia incompleta: la lucha de los Mexicanos por el voto en el exterior". In: Calderón Chelius, Leticia (eds.). Votar en la distancia : la extensión de los derechos políticos a migrantes, experiencias comparadas. México: Instituto Mora, 2004. P. 217-267.

COVE. "Informe final sobre el voto en el extranjero". (2006). [Consultada el Access 2006] http://mxvote06.ife.org.mx/libro blanco/.

de la Garza, Rodolfo 0.; Desipio, Louis. "Interests Not Passions: MexicanAmerican Attitudes Toward Mexico and Issues Shaping U.S.-Mexico Relations". International Migration Review. Vol. 32. No. 2 (1998). P. 401-422.

Delano, Alexandra. "From Limited to Active Engagement: Mexico's Emigration Policies from a Foreign Policy Perspective (2000-2006)". International Migration Review. Vol. 43. No. 4 (2009). P. 764-814.

Escobar, Cristina. "Voto colombiano". (2011). [Consultada el Access 2011]

Espinoza Valle, Víctor Alejandro. El voto lejano : cultura politíca y migración México-Estado Unidos. Mexico: Colegio de la Frontera Norte, 2004. P.

Geys, Benny. "Explaining voter turnout: A review of aggregate-level research". Electoral Studies. Vol. 25. No. 4 (2006). P. 637-663.

Goldring, Luin. "From Market Membership to Transnational Citizenship: The Changing Politization of Transnational Social Spaces". L'Ordinaire LatinoAméricain. Vol. No. 173-174 (1998). P. 167-172.

Gómez Quiñonez, Juan. "Notes on an interpretation of the relations between the Mexican community in the United States and Mexico ". In: Vázquez, Carlos; García y Griego, Manuel (eds.). Mexican-US Relations. Conflict and Convergence. Los Angeles:: UCLA Chicano Studies Research Center/UCLA Latin American Center., 1983. P. 399-416.

IFE. "Informe final de la Comisión de especialistas que estudia las modalidades del voto de los mexicanos en el extranjero". (1998). [Consultada el http://www.ife.org.mx/documentos/AI/indve.htm.

IME. "Mexicanos Residentes en el Mundo 2009". (2010). [Consultada el Access 2010]

Lafleur, Jean-Michel. Le transnationalisme politique. Pouvoir des communautés immigrées dans leurs pays d'accueil et d'origine. Louvain-la-Neuve: AcademiaBruylant, 2005. P. 76.

Lafleur, Jean-Michel. Diaspora y voto en la distancia: La participacion politica transnacional del emigrante boliviano en las elecciones présidenciales del 2009. Barcelona: CIDOB, 2012. P.

Lafleur, Jean-Michel; Calderón Chelius, Leticia. "Assessing Emigrant Participation in Home Country Elections: The Case of Mexico's 2006 Presidential Election". International Migration. Vol. 49. No. 3 (2011). P. 99-124. 
Leiken, Robert S. The Melting Border : Mexico and Mexican Communities in the United States. Washington: Center for Equal Opportunity, 2000. P.

Marcelli, E.A.; Cornelius, W.A. "Immigrant Voting in Home-country Elections: Potential Consequences of Extending the Franchise to Expatriate Mexicans Residing in the United States". Mexican Studies/Estudios Mexicanos. Vol. 21. No. 3 (2005). P. 429-460.

Martínez Saldaña, J. "In Search of our Lost Citizenship: Mexican Immigrants, the Right to Vote, and the Transition to Democracy in Mexico". L'Ordinaire LatinoAméricain. Vol. No. 173-174 (1998). P. 153-166.

McCann, James; Cornelius, W.A.; Leal, David. "Mexico's 2006 Voto Remoto and the Potential for Transnational Civic Engagement among Mexican Expatriates". (2006). [Consultada el Access 2006]

Moctezuma Longoria, Miguel. "La voz de los actores. Ley migrante y Zacatecas". Migración y Desarrollo. Vol. No. 1 (2003). P. 100-118.

Østergaard-Nielsen, Eva. Transnational Politics. Turks and Kurds in Germany. London: Routledge, 2003. P.

Roberts, Bryan; Frank, Reanne; Lozano-Ascencio, Fernando. "Transnational Migrant Communities and Mexican Migration to the US". Ethnic and Racial Studies. Vol. 22. No. 2 (1999). P. 238-266.

Santamaría Gomez, Arturo. "Politics without Borders or Postmodern Nationality: Mexican Immigration to the United States". Latin American perspectives. Vol. 30. No. 2 (2003). P. 274-86.

Santamaría Gómez, Arturo. "El movimiento vasconcelista en Estados Unidos y los orígenes del voto mexicano en el extranjero ". In: Santamaría Gómez, Arturo (eds.). Mexicanos en Estados Unidos: La nación, la política y el voto sin fronteras. Culiacan and Mexico: UAS and PRD, 2001. P. 127-145.

Sepúlveda, Graciela G. "Demandas de participacion politica de los Mexicanos en el estado de California, EUA. La lucha desde afuera". In: Ramírez Paredes, Gustavo (eds.). El sistema politico mexicano visto por los mexicanos de afuera. El caso de la Comunidad Chicana en el Estado de California. Mexico: UNAM, 1991. P. 45-62.

Smith, Peter H. "El imperio del PRI". In: Anna, Timothy; Bazant, Jan; Katz, Friedrich; Womack, John; Meyer, Jean; Knight, Alan; Smith, Peter H. (eds.). Historia de México. Barcelona: Crítica, 2003. P. 321-384.

Smith, Robert C. "Contradictions of Diasporic Institutionalization in Mexican Politics: The 2006 Migrant Vote and Other Measures of Inclusion". Ethnic and Racial Studies. Vol. 31. No. 4 (2008). P. 708-741.

Smith, Tony. Foreign Attachments -The Power of Ethnic Groups in the Making of American Foreign Policy. Cambridge: Harvard University Press, 2000. P. 224.

Suro, Roberto; Escobar, Gabriel. "Survey of Mexicans Living in the U.S. on Absentee Voting in Mexican Elections". (2006). [Consultada el Access 2006] http://pewhispanic.org/files/reports/60.pdf.

Turner, Simon. "The waxing and waning of the political field in Burundi and its diaspora ". Ethnic and Racial Studies. Vol. 31. No. 4 (2008). P. 742-765.

Waldinger, Roger. "A Limited Engagement: Mexico and its Diaspora". (2008). [Consultada el Access 2008] 
' Jusqu'en 1996, le droit de vote de la diaspora est rendu impossible par l'article 36 de la Constitution selon lequel le vote s'exerce nécessairement dans la circonscription électorale à laquelle le citoyen appartient.

ii L'usage du terme de "pocho" s'est développé durant le régime du PRI pour désigner les émigrés de façon dénigrante. 